\title{
NEAR-INFRARED SPECTROSCOPY OF SN 2017EAW IN 2017: CARBON MONOXIDE AND DUST FORMATION IN A TYPE II-P SUPERNOVA
}

\author{
J. Rho ${ }^{1,2}$, T. R. Geballe ${ }^{3}$, D. P. K. Banerjee ${ }^{4}$, L. Dessart $^{5}$, A. Evans ${ }^{6}$, And V. Joshi ${ }^{4}$ \\ Draft version July 31, 2018
}

\begin{abstract}
The origin of dust in the early Universe has been the subject of considerable debate. Core-collapse supernovae (ccSNe), which occur several million years after their massive progenitors form, could be a major source of that dust, as in the local universe several ccSNe have been observed to be copious dust producers. Here we report nine near-infrared $(0.8-2.5 \mu \mathrm{m})$ spectra of the Type II-P SN 2017eaw in NGC 6946, spanning the time interval $22-205$ days after discovery. The specta show the onset of $\mathrm{CO}$ formation and continuum emission at wavelengths greater than $2.1 \mu \mathrm{m}$ from newly-formed hot dust, in addition to numerous lines of hydrogen and metals, which reveal the change in ionization as the density of much of the ejecta decreases. The observed CO masses estimated from an LTE model are typically $10^{-4} \mathrm{M}_{\odot}$ during days $124-205$, but could be an order of magnitude larger if non-LTE conditions are present in the emitting region. The timing of the appearance of CO is remarkably consistent with chemically controlled dust models of Sarangi \& Cherchneff.
\end{abstract}

Subject headings: molecular processes - ISM:molecules - supernova remnants: ISM:dust - Supernovae: individual: SN 2017eaw

\section{INTRODUCTION}

Whether core-collapse supernovae (ccSNe) are a significant source of dust in the Universe is a long-standing question. The large quantities of dust observed in highredshift galaxies (e.g. Isaak et al. 2002; Watson et al. 2015) raise a fundamental question as to the cause of its early presence, because stars, which are thought to produce most of the interstellar dust when on the AGB in the modern Milky Way and nearby galaxies, could not have evolved to the dust-producing stage in very high-redshift galaxies. In contrast, $\mathrm{SNe}$ from massive stars occur just millions of years after they form. Michalowski (2015) concluded that the early appearance of dust could be explained if ccSNe do not subsequently destroy the dust they create. However, dust forms and grows in the interstellar medium (Spitzer 1978; Zhukovska et al. 2018), and the dust in very high- $z$ systems might be produced primarily there, seeded by heavy elements and/or surviving dust from $\mathrm{SNe}$ (Draine 2009). Depending on the progenitor mass, dust masses of $0.1-1.0 \mathrm{M}_{\odot}$ per ccSN (Todini \& Ferrara 2001; Nozawa et al. 2003) can account for the amount of dust observed in very high-redshift galaxies. Evidence that supernova-produced dust is the primary source of dust in the early Universe is still scarce (see discussion in Gall et al. 2011; Rho et al. 2018, and references therein) and requires further study.

\footnotetext{
${ }^{1}$ SETI Institute, 189 N. Bernardo Ave., Mountain View, CA 94043; jrho@seti.org

2 Stratospheric Observatory for Infrared Astronomy, NASA Ames Research Center, MS 211-3, Moffett Field, CA 94035

3 Gemini Observatory, 670 N. Aohoku Place, Hilo, HI, 96720, USA

${ }^{4}$ Physical Research Laboratory, Navrangpura, Ahmedabad, Gujarat 380009, India

${ }^{5}$ Unidad Mixta Internacional Franco-Chilena de Astronomia (CNRS UMI 3386), Departamento de Astronomia, Universidad de Chile, Camino El Observatorio 1515, Las Condes, Santiago, Chile

${ }^{6}$ Astrophysics Group, Keele University, Keele, Staffordshire, ST5 5BG, UK
}

Spitzer mapping of the young supernova remnant (YSNR) Cas A revealed a remarkable similarity between the dust and $\mathrm{CO}$ distributions, confirming that dust forms and is preserved in at least some ccSNe ejecta (Rho et al. 2008, 2012). Four YSNRs including SN 1987A (Matsuura et al. 2011, 2015), the Crab Nebula (Gomez et al. 2012; Temim et al. 2012; Temim \& Dwek 2013), Cas A (Rho et al. 2008; De Looze et al. 2017) and G54.1+0.3 (Temim et al. 2017; Rho et al. 2018) have current dust masses of 0.1-0.9 $\mathrm{M}_{\odot}$, consistent with theoretical models (Nozawa et al. 2003; Todini \& Ferrara 2001). The results suggest that some ccSNe are dust factories, and thus that they could be very important at high $z$, when other significant dust production processes may not exist. However, the fraction of ccSNe that produce this much dust is not known. Observing dust in additional local ccSNe and following its production and evolution in individual $\mathrm{SNe}$ over an extended period of time will help provide the answer.

Current understanding of dust chemistry and composition in ccSNe is limited. Some models (e.g. Nozawa et al. 2003) suggest that dust forms $350-900$ days after a ccSN explosion, with carbon dust being one of the first condensates. Those models assume that little carbon is locked up in carbon monoxide (CO) and almost all of it is available for dust formation. $\mathrm{CO}$ is one of the most powerful coolants in the ejecta of type II SNe and, once it forms at several thousand $\mathrm{K}$ in some portions of the ejecta, is believed to be responsible in large part for cooling those ejecta to temperatures $\lesssim 1700 \mathrm{~K}$ at which dust can form. Dust evolution models indicate that dust production depends significantly on the $\mathrm{C} / \mathrm{O}$ ratio in the gas (Morgan \& Edmunds 2003; Dwek et al. 1998), as well as on the rate of destruction of CO, by impact both with the energetic electrons produced by the radioactive decay of ${ }^{56} \mathrm{Co}$ and with $\mathrm{He}^{+}$(Cherchneff \& Dwek 2010). Measurements of newly formed CO can shed light on the dust production rate.

Direct physical association of dust in high- $z$ galaxies 
TABLE 1

Summary of Observations and Results of CO Modeling

\begin{tabular}{cccccccc}
\hline \hline (yyyymmdd) & Day & $\begin{array}{c}\text { exposure } \\
(\mathrm{sec})\end{array}$ & $\begin{array}{c}\text { CO mass } \\
\left(10^{-4} \mathrm{M} \odot\right)\end{array}$ & $\begin{array}{c}T^{\mathrm{a}} \\
(\mathrm{K})\end{array}$ & $\begin{array}{c}\mathrm{FWHM}^{b} \\
\left(\mathrm{~km} \mathrm{~s}^{-1}\right)\end{array}$ & $\begin{array}{c}J, H, K s^{c} \\
(\mathrm{mag})\end{array}$ & $\begin{array}{c}\text { scale }^{d} \\
\text { factor }^{c}\end{array}$ \\
\hline 20170605 & 22 & 240 & & $\ldots$ & $\ldots$ & $\ldots$ & 1.000 \\
20170616 & 33 & 360 & $\ldots$ & $\ldots$ & $\ldots$ & $11.61 \pm 0.04,11.39 \pm 0.05,11.16 \pm 0.04$ & 0.505 \\
20170626 & 43 & 360 & $\ldots$ & $\ldots$ & $\ldots$ & $\ldots$ & 0.218 \\
20170706 & 53 & 360 & $\ldots$ & $\ldots$ & $\ldots$ & $\ldots$ & 0.112 \\
20170829 & 107 & 600 & $\leq 0.4$ & $\ldots$ & $\ldots$ & $\ldots$ & 0.070 \\
20170915 & 124 & 600 & $0.6-1.6$ & 3000 & 2800 & $\ldots$ & 0.051 \\
20171001 & 140 & 1200 & $1.0-1.9$ & 3300 & 2850 & $\ldots .012$ \\
20171030 & 169 & 1200 & $1.6-2.2$ & 3000 & 2850 & $13.65 \pm 0.06,13.45 \pm 0.08,13.31 \pm 0.10$ & 0.005 \\
20171205 & 205 & 1080 & $1.9-2.2$ & 2700 & 2750 & $14.08 \pm 0.07,14.02 \pm 0.10,13.98 \pm 0.12^{e}$ & 0.002 \\
\hline \hline
\end{tabular}

${ }^{\mathrm{a}} \sigma \sim 200 \mathrm{~K} ;{ }^{b} \sigma \sim 200 \mathrm{~km} \mathrm{~s}^{-1} ;{ }^{c}$ Photometry obtained by one of us (VJ) at 1.2-meter Mount Abu Infrared Telescope, India.; ${ }^{d}$ Spectra in Fig. 1 have been multiplied by these values.; ${ }^{e}$ The JHK imaging observations were taken on 2017 November 30.

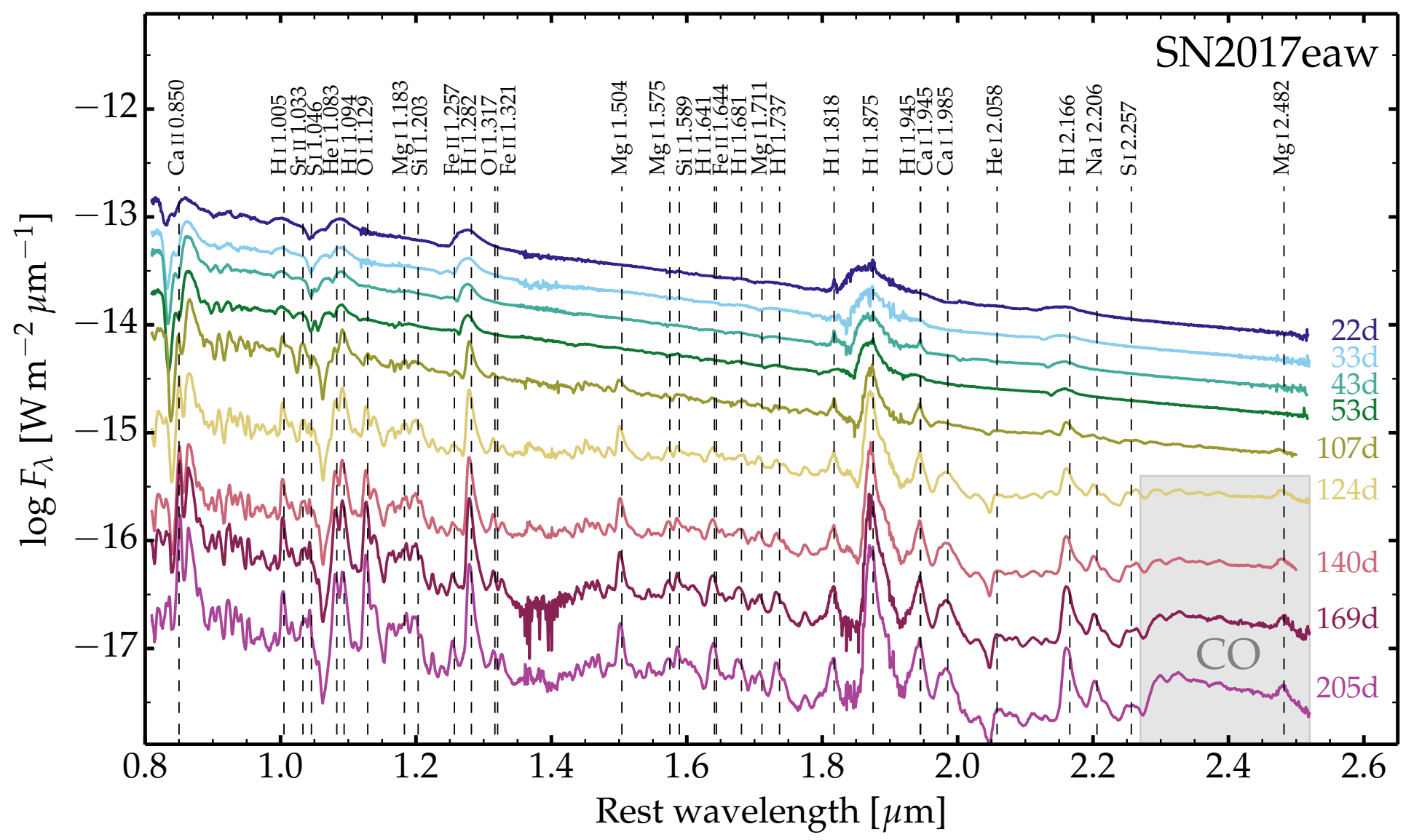

FIG. 1.- Gemini GNIRS $0.81-2.52 \mu \mathrm{m}$ spectra (dereddened) of SN 2017eaw obtained in 2017, in time order (top to bottom). Plotted spectra multiplied by values in Table 1. Prominent and important lines are labeled. Wavelength interval with overtone CO emission is shaded.

with high- $z$ ccSNe is currently not possible, and thus to test the idea one must study dust production in local ccSNe. On 2017 May 14, a bright ccSN, 2017eaw, was discovered in the starburst galaxy NGC 6946. It has been classified as Type II-P (ATel 10376; Xiang et al. 2017). The progenitor is known to be a red supergiant (RSG; ATel 10378; van Dyk et al. 2017; Kilpatrick \& Foley 2018). Because of its proximity and location in the sky, SN 2017eaw is ideal for studying the onset and evolu- tion of both molecule and dust formation from a northern hemisphere telescope. Here we present and describe nine Gemini near-infrared spectra obtained during 2017 JuneDecember and discuss the evolution of the spectrum, the detection of hot $\mathrm{CO}$ and an estimate of its mass, and emission from newly-formed dust. ATel 10765 (Rho et al. 2017) is a brief report on the first six of these spectra. Estimates of the distance to NGC 6946 vary from 4 to $12.7 \mathrm{Mpc}$ in the NASA/IPAC Extragalactic Database 


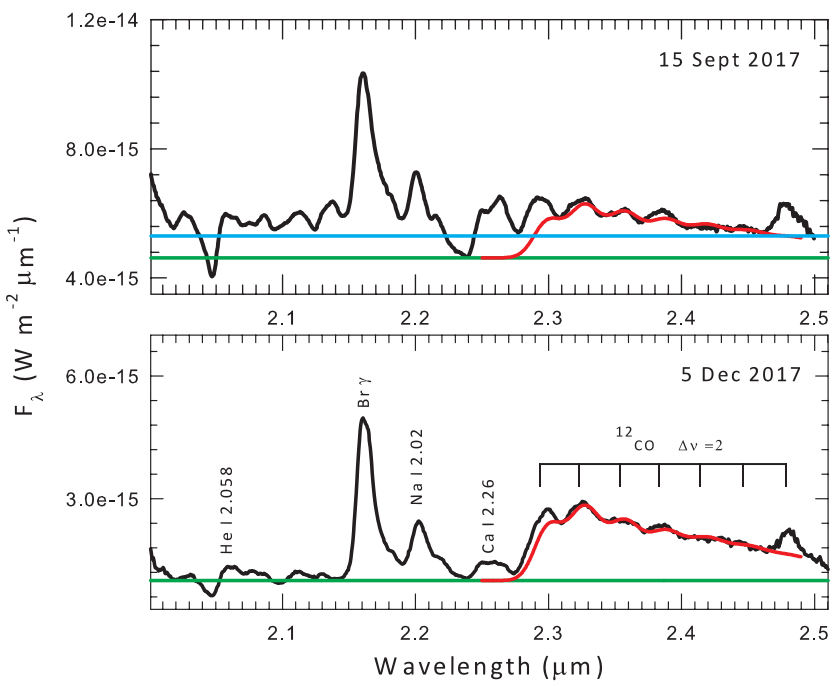

Fig. 2.- Zoomed in CO first overtone band emission superposed on LTE model fits (red lines) at $124 \mathrm{~d}$ and $205 \mathrm{~d}$. Observed spectra in black. Green and blue lines in uppermost panel are possible continua. Parameters of fits are in Table 1.

(NED). We adopt $7.72 \pm 0.32 \mathrm{Mpc}$ from Anand et al. (2018).

\section{OBSERVATIONS AND DATA REDUCTION}

We obtained 0.81-2.52 $\mu \mathrm{m}$ spectra of SN 2017eaw using the Gemini Near-Infrared Spectrograph (GNIRS) on the 8.1-meter Frederick C. Gillett Gemini-North telescope, for programs GN-2017A-DD-8 and GN-2017BDD-11. The observing dates and exposure times are listed in Table 1. GNIRS was configured in its crossdispersed mode, using its 32 line/ $\mathrm{mm}$ grating and a 0.45 arcsecond-wide slit to provide a resolving power of $\sim 1200$ $\left(250 \mathrm{~km} \mathrm{~s}^{-1}\right)$. Observations were made in the standard stare / nod-along-slit mode with a nod angle of 3 arcseconds. Nearby early A-type dwarfs, observed either just before or just after SN 2017eaw to minimize differences in airmass, served as telluric and flux standards. Although the key questions we wished to address required only Kband spectra, and in particular the longer wavelength portion of that band, where hot newly formed dust and $\mathrm{CO}$ are both expected to emit, the full spectral coverage provides a wealth of information regarding the evolution of the kinematics, ionization state, and elemental abundances.

Data reduction utilized both the GNIRS crossdispersed reduction pipeline and manual, order-by-order reduction for the shortest wavelength orders and for instances in other orders where the pipeline appeared to produce spurious results. The manual reduction used standard IRAF ${ }^{7}$ and Figaro tools for flatfielding, spike removal, rectification of spectral images, extraction, wavelength calibration, and removal of hydrogen absorption lines in the spectra of the standard stars. When order-by-order reduction was used, spectral segments covering different orders were stitched together after small scaling factors were applied, to produce final continuous spectra between $0.81-2.52 \mu \mathrm{m}$. The reli-

7 IRAF is distributed by the National Optical Astronomy Observatories, which are operated by the Association of Universities for Research in Astronomy, Inc., under cooperative agreement with the National Science Foundation.

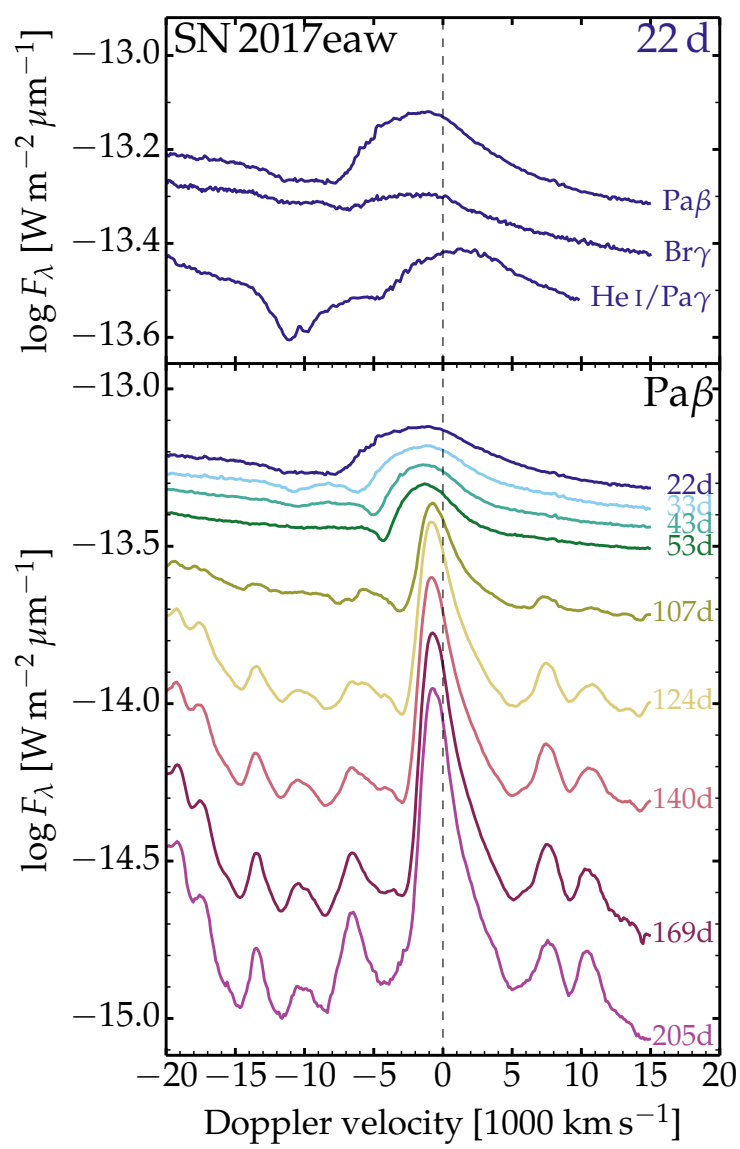

FIG. 3.- Upper panel: velocity profiles of hydrgen $\mathrm{Pa} \beta$ and $\mathrm{Br}$ $\gamma$ and blended $\mathrm{H} / \mathrm{He} \mathrm{Pa} \gamma / 1.083 \mu \mathrm{m}$ on day 22 . Doppler velocity relative to $\mathrm{H}$ i lines. Lower panel: evolution of the $\mathrm{Pa} \beta$ and $\mathrm{Br} \gamma$ line profiles.

ability of the spectra in the two intervals from 1.35 to $1.45 \mu \mathrm{m}$ and from 1.80 to $1.95 \mu \mathrm{m}$ is low because of low atmospheric transmission; one should view the spectra at these wavelengths with caution. In several of the spectra that were reduced manually the $\mathrm{H}_{\mathrm{I}}$ lines in the telluric standard in the $1.80-1.95 \mu \mathrm{m}$ interval were not removed prior to ratioing. At most other wavelengths, although the signal-to-noise ratio varies considerably, it is very high judging by the repetitiveness of the weaker features in spectra obtained on adjacent dates. Because some narrow-slit spectra were obtained in conditions of poor seeing and slightly non-photometric skies, flux densities coud be uncertain by $\pm 30 \%$.

\section{OVERVIEW OF SPECTRA}

Figure 1 shows the nine $0.8-2.5 \mu \mathrm{m}$ spectra of $\mathrm{SN}$ 2017eaw obtained during 2017. The earliest spectrum was obtained 22 days after discovery, the latest at 205 days. These data form the most extensive set of nearinfrared spectra of a Type II-P supernova obtained to date. We are making them available to the astronomy community via WISEREP ${ }^{8}$.

The first four spectra, obtained during the photo-

8 https://wiserep.weizmann.ac.il/ 
spheric phase, are dominated by hydrogen recombination line emission and the Ca II triplet at $0.85 \mu \mathrm{m}$, all superimposed on a continuum that decreases monotonically with increasing wavelength. The spectrum at day 107 marks a transition, as part of the ejecta begins to turn nebular in density and numerous neutral atomic lines begin to appear across the entire wavelength range. According to the optical photometry of Tsvetkov et al. (2018), SN 2017eaw turned nebular (i.e., the nebular brightness follows the $0.01 \mathrm{mag} \mathrm{d}^{-1}$ decay rate for ${ }^{56} \mathrm{Co}$ ) at an age of $\sim 125 \mathrm{~d}$. At $124 \mathrm{~d}$ the atomic lines in the near-IR spectra have become much more prominent, largely because the continuum has greatly decreased.

Spectral signatures of several CO overtone bands at 2.3-2.5 $\mu \mathrm{m}$ are evident in emission in the $124 \mathrm{~d}$ spectrum and afterward (see Figures 1 and 2), although the cuton of the $2-0$ CO emission near $\sim 2.29 \mu \mathrm{m}$ is not yet obvious until $169 \mathrm{~d}$. There is marginal evidence for weak CO emission earlier, at day 107. In addition, beginning on day 124 there is a flattening of the continuum at $\lambda>$ $2.1 \mu \mathrm{m}$, not seen in the earlier spectra, which we interpret as emission from newly formed hot dust. The flattening of the continuum beyond $2.1 \mu \mathrm{m}$ is also present in the final three spectra.

\section{ANALYSIS AND DISCUSSION}

\subsection{Evolution of the Atomic Line Spectrum}

During the photospheric phase, in addition to the $\mathrm{HI}$ and $\mathrm{Ca}$ II lines mentioned above, there are contributions from $\mathrm{C}_{\mathrm{I}}, \mathrm{Mg} \mathrm{I}, \mathrm{Mg}$ II, and later S I (see Fig. 1). Initially, the $\mathrm{H}$ I lines have $\mathrm{P}$ Cygni profiles and full extents of many thousands of $\mathrm{km} \mathrm{s}^{-1}$ but the latter decrease monotonically with time as shown in Figure 3. The extremely broad and shallow blue-shifted absorption troughs at the $\mathrm{H}$ I $\mathrm{Pa} \gamma, \mathrm{Pa} \beta$, and $\mathrm{Br} \gamma$ ines in the early spectra may be the result of sustained absorption at high velocity by He I, which results from an ionisation freeze-out of He in the outer ejecta (Dessart \& Hillier 2008). Absorption by He I lines at $1.083,1.253,1.278,2.113$, and $2.165 \mu \mathrm{m}$ may contribute to these extended troughs.

In the observed nebular phase, the SN luminosity has dropped by two magnitudes since the photospheric phase, and a much higher fraction of the ${ }^{56}$ Co decay power emerges in lines. The nebular spectrum forms in the regions that absorb this decay power, located in what used to be the He core and the base of the H-rich envelope. While the lines present in the photospheric phase (i.e. from $\mathrm{H}$ I, HeI, CaII) persist, additional lines from intermediate mass elements (O I, Na I, Mg I, Si I, S I, and $\mathrm{CaI}$ ), and iron-group elements (primarily Fe I and Fe II). Sr II $1.033 \mu \mathrm{m}$ are observed (see Fig. 1), especially around the transition to the nebular phase. The line profiles in the nebular phase are narrower than earlier and the peaks in some of lines are blue-shifted by several hundreds of $\mathrm{km} \mathrm{s}^{-1}$ (Fig. 3). Early formation of dust, as in the case of SN2010ji which has similar velocity shifts (Gall et al. 2014) could be the cause of this; two other possibile explanations are continuum opacity of the ejected gas and asymmetric ejecta.

We have generated a selection of simulations from Dessart et al. (2013) and Dessart et al. (2018) for representative Type II SNe from $15 \mathrm{M}_{\odot}$ progenitors, exploded to yield ejecta masses of $12.5 \mathrm{M}_{\odot}$ with $1.2 \times 10^{51} \mathrm{erg}$. For

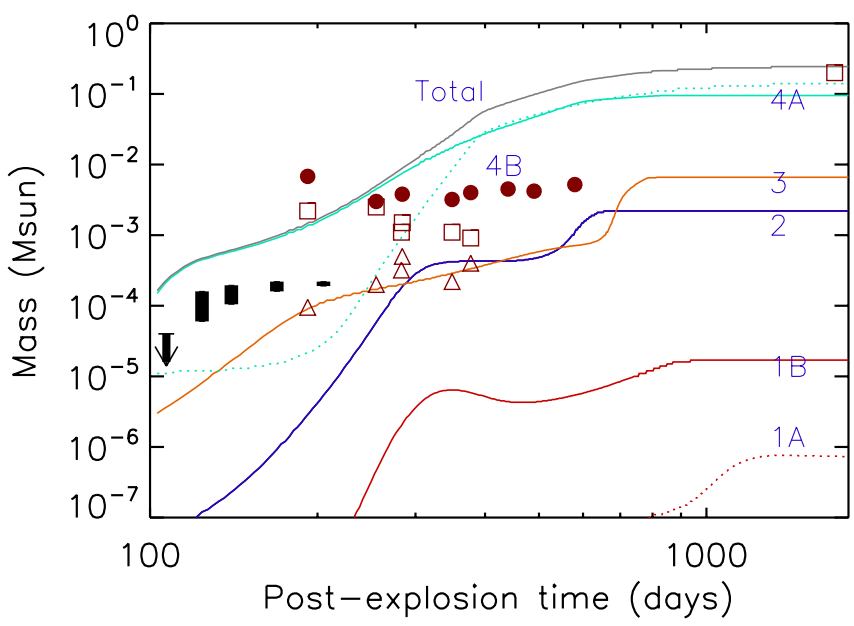

Fig. 4.- CO masses of SN 2017eaw (black, filled rectangles) plotted on model of $\mathrm{CO}$ produced by a $15 \mathrm{M}_{\odot}$ progenitor (Sarangi \& Cherchneff 2013), for ejecta zones from innermost (1A) to outermost (4B). Vertical extents of black rectangles denote ranges of estimated CO masses, which depend on choice of 2.3-2.5- $\mu \mathrm{m}$ continuum. The zones include $\mathrm{Si} / \mathrm{S} / \mathrm{Fe}-$ rich (zone 1), O/Si/S-rich (zone 2), $\mathrm{O} / \mathrm{Mg} /$ Si-rich (zone 3), O/C/Mg-rich (zone 4) (Cherchneff \& Dwek 2009). Zones 1 and 4 are subdivided into A and B subzones (see Table 1 of Sarangi \& Cherchneff 2013). The grey line represents the mass summed over all zones. The largest amounts of CO primarily form in zones 4A and 4B. CO masses for SN 1987A derived for LTE (small triangles) and non-LTE (small squares) from models of Liu et al. (1992) and small circles for thermal chemical model of Liu \& Dalgarno (1995) are also plotted.

the photospheric phase the models are good matches to the observations. For example, the spectrum at $43 \mathrm{~d}$ corresponds to a model of a red supergiant explosion (model m15mlt3 from Dessart et al. 2013) with a photospheric temperature of $5450 \mathrm{~K}$ and a photospheric velocity of $4900 \mathrm{~km} \mathrm{~s}^{-1}$. In the nebular phase, the overall agreement of models of blue supergiant explosion (model Bsm from Dessart et al. 2018) endowed with $0.084 \mathrm{M}_{\odot}$ of ${ }^{56} \mathrm{Ni}$ and the spectra is satisfactory, although the models overestimate the strengths of $\mathrm{Mg}$ I lines and underestimate the strengths of $\mathrm{C}$ I lines around $1 \mu \mathrm{m}$. The apparent shallow continuum "bump" observed at 1.40-1.75 $\mu \mathrm{m}$ (see Fig. 1) is due at least in part to numerous overlapping lines, largely from Fe I and Fe II.

\section{2. $C O$ and Dust}

Carbon monoxide is first securely detected at $124 \mathrm{~d}$. We have estimated the CO mass at days 124, 140, 169 and 205 using the LTE model developed by Das et al. (2009) and used by Banerjee et al. (2016), Joshi et al. (2017), and others. Two examples are shown in Figure 2. The model assumes pure ${ }^{12} \mathrm{C}^{16} \mathrm{O}$ and optically thin, LTE emission. The presence of $\mathrm{CO}$ in each of the spectra is clear, based on the close match of peaks in the spectrum to the wavelengths of the $3-1,4-2$, and $5-3$ band heads $(2.323,2.351$, and $2.383 \mu \mathrm{m}$, respectively), the fair match to the $2-0$ band head at $2.294 \mu \mathrm{m}$ (which may be contaminated by an atomic line, probably $\mathrm{C}_{\mathrm{I}}$ $2.291 \mu \mathrm{m}$ ) and, especially in the final two spectra, the large rise in flux density near the wavelength of the 2-0 band head. However, the strength of the CO emission and the $\mathrm{CO}$ mass derived from it are uncertain, due to the uncertainty in the location of the K-band continuum, 
especially on days 124 and 140 . For self-consistency, in each of the final four 2017 spectra we initially adopted a flat continuum (due to the photosphere and presumed dust emission) passing through the bottom of the trough at $2.23 \mu \mathrm{m}$. In doing so we essentially assumed that atomic line emission accounts for the elevated "pseudocontinuum" at $2.0-2.3 \mu \mathrm{m}$ on those two dates, but does not contribute significantly at longer wavelengths where the $\mathrm{CO}$ emits. However, it is quite possible that these minima are $\mathrm{P}$ Cygni troughs. If so, the above choice results in significant overestimates of the CO mass on days 124 and 140, but not on days 169 and 205, as on those dates the "pseudo-continuum" is much weaker than the $\mathrm{CO}$ emission. Thus, the upper limits to the derived CO masses listed in Table 1 for $124 \mathrm{~d}$ and $140 \mathrm{~d}$ should be regarded with caution. For example, if the adopted continuum in the top panel of Figure 2 is the blue line, the $\mathrm{CO}$ emission strength on that date would be $\sim 3$ times lower than that limit. The range of values of the $\mathrm{CO}$ mass in Table 1 reflect this uncertainty.

Best fit model spectra were determined by varying the CO mass, temperature, and velocity disperson with the goal of minimizing the reduced $\chi^{2}$ value over the region 2.30 to $2.46 \mu \mathrm{m}$ (thus excluding the $2-0$ band head and the strong $[\mathrm{Mg} \mathrm{I}]$ line at $2.48 \mu \mathrm{m})$. The fit results are summarized in columns 4-6 of Table 1 . We obtain CO masses of $\sim 1 \times 10^{-4} \mathrm{M}_{\odot}$ on all four dates from the LTE model and the assumed continua. The CO mass depends on the distance, $M_{7.72}\left(d / d_{7.72}\right)^{2}$ where $M_{7.72}$ (in Table 1) and $d_{7.72}$ are the mass and distance assuming a distance of $7.72 \mathrm{Mpc}$, and $d$ is an actual distance to SN2017eaw. The masses on the first two dates may be considerably overestimated, as discussed above. On the other hand, if the molecular gas is not in LTE, the CO masses could be an order of magnitude larger, in analogy to the models of Liu et al. (1992) for SN 1987A. The best fit CO temperatures and velocity dispersions for all four spectra are typically $3000 \mathrm{~K}$ and $2800 \mathrm{~km} \mathrm{~s}^{-1}$, respectively.

Observations of $\mathrm{CO}$ in $\mathrm{SNe}$ are fairly rare; detections from about a dozen SNe, including a Type Ic (Hunter et al. 2009), are known and summarized in Sarangi et al. (2018). The evolution of the CO emission in ccSNe has only been studied over a lengthy time interval for SN 1987A (SNII-pec, a rare class but close to the Type II-P $\mathrm{SN})$. In Figure 4 the estimated CO masses of SN 2017eaw are compared to those of SN 1987A and to the CO mass as a function of time predicted by Sarangi \& Cherchneff (2013). In both objects overtone CO emission is detected for the first time shortly after 100 days: 112 days for SN 1987A (Spyromilio et al. 1988) and at $124 \mathrm{~d}$ for SN 2017 eaw (implying significant CO production began between $107 \mathrm{~d}$ and $124 \mathrm{~d}$ ). These dates are remarkably consistent with the chemically controlled dust models by Sarangi \& Cherchneff $(2013,2015)$, in which the first appearance of $\mathrm{CO}$ is predicted to be at $\sim 100$ days. The $\mathrm{CO}$ mass observed in SN 2017eaw $\left(\sim 10^{-4} \mathrm{M}_{\odot}\right)$ is also roughly comparable to amounts in the models, in which most of the $\mathrm{CO}$ is produced in zones $4 \mathrm{~A}$ and $4 \mathrm{~B}$ (Sarangi \& Cherchneff 2013). Individually each represents less than $0.1 \%$ of the available $\mathrm{C}$ and $\mathrm{O}$ masses $\left(0.1 \mathrm{M}_{\odot}\right.$ and $1.0 \mathrm{M}_{\odot}$ respectively, based on the models described above). However, $\mathrm{CO}$ may continue to form during the first few or a few ten years after the explosion and thus may result in a vastly larger amount of $\mathrm{CO}$ than observed to be emitting in the first overtone band at any one time.

We have interpreted the flattening of the K-band continuum starting at $2.1 \mu \mathrm{m}$, in the four GNIRS spectra obtained from $124 \mathrm{~d}$ to $205 \mathrm{~d}$, to be due to emission from hot newly condensed dust. When we fit the continua of the spectra above $2.1 \mu \mathrm{m}$ using carbon dust, dust temperatures of $\sim 1400 \mathrm{~K}$ and $\sim 1200 \mathrm{~K}$ are consistent with the spectra at $124 \mathrm{~d}$ and $205 \mathrm{~d}$, respectively. We suggest that the carbon dust formed in SN2017eaw is graphitic carbon because it condenses at $1150-1690 \mathrm{~K}$, whereas amorphous carbon condenses at $\lesssim 1100 \mathrm{~K}$ (Fedkin et al. 2010; Ebel 2000). Dust formation at $124 \mathrm{~d}$ is much earlier than predicted by some models (Todini \& Ferrara 2001; Nozawa et al. 2003). However, the models of Schneider et al. (2004) and Sarangi \& Cherchneff (2015), and Lazzati and Heger (2015) suggest that dust formation begins at $\sim 150 \mathrm{~d}$ and continues until $2000 \mathrm{~d}$. Recent observations show that dust formed at during $40-868 \mathrm{~d}$ in SN2010jl (Gall et al. 2014) and continues to be present in SN1987A as late as 25 yr after outburst (Matsuura et al. 2011; Indebetouw et al. 2014; Zanardo et al. 2014).

The early sign of dust formation in SN2017eaw appears to be supported by an excess of 3.6- $\mu \mathrm{m}$ emission seen on day 193 in Spitzer IRAC observations (S. Tinyanont et al. 2018, private communication). This work serves as an early alert for further monitoring of the formation/evolution of the dust and $\mathrm{CO}$ in this object, particularly in the infrared.

As of the date of the final spectrum (UT 2017 December 5), the rate of $\mathrm{CO}$ production remained strong. Because of this, we plan to obtain additional near-IR measurements of SN 2017eaw during 2018.

This paper is based on observations obtained at the Gemini Observatory, which is operated by the Association of Universities for Research in Astronomy, Inc., under a cooperative agreement with the NSF on behalf of the Gemini partnership: the National Science Foundation (United States), the National Research Council (Canada), CONICYT (Chile), Ministerio de Ciencia, Tecnologa e Innovacin Productiva (Argentina), and Ministrio da Cincia, Tecnologia e Inovao (Brazil). JR acknowledges support in part for this work from NASA ADAP grant (NNX12AG97G). Research at the Physical Research Laboratory is funded by the Department of Space, Government of India. We thank Dr. Arka Sarangi for providing his models, and the anonymous referee for insightful comments.

\section{REFERENCES}

Anand, G. S., Rizzi, L., \& Tully, R. B. 2018, ArXiv e-prints, arXiv:1807.05229

Banerjee, D. P. K., Srivastava, M. K., Ashok, N. M., \& Venkataraman, V. 2016, MNRAS, 455, L109

Cherchneff, I., \& Dwek, E. 2009, ApJ, 703, 642
Das, R. K., Banerjee, D. P. K., \& Ashok, N. M. 2009, MNRAS, 398,375

De Looze, I., Barlow, M. J., Swinyard, B. M., et al. 2017,

MNRAS, 465, 3309

Dessart, L., \& Hillier, D. J. 2008, MNRAS, arXiv:0710.0784 
Dessart, L., Hillier, D. J., Waldman, R., \& Livne, E. 2013, MNRAS, 433, 1745

Dessart, L., Hillier, D. J., \& Wilk, K. D. 2018, submitted to A\&A

Draine, B. T. 2009, in Astronomical Society of the Pacific

Conference Series, Vol. 414, Cosmic Dust - Near and Far, ed. T. Henning, E. Grün, \& J. Steinacker, 453

Ebel, D. S. 2000, J. Geophys. Res., 105, 10363

Fedkin, A. V., Meyer, B. S., \& Grossman, L. 2010, Geochim. Cosmochim. Acta, 74, 3642

Gall, C., Hjorth, J., \& Andersen, A. C. 2011, A\&A Rev., 19, 43

Gall, C., Hjorth, J., Watson, D., et al. 2014, Nature, 511, 326

Gomez, H. L., Krause, O., Barlow, M. J., et al. 2012, ApJ, 760, 96

Hunter, D. J., Valenti, S., Kotak, R., et al. 2009, A\&A, 508, 371

Indebetouw, R., Matsuura, M., Dwek, E., et al. 2014, ApJ, 782, L2

Isaak, K. G., Priddey, R. S., McMahon, R. G., et al. 2002, MNRAS, 329, 149

Joshi, V., Banerjee, D. P. K., \& Srivastava, M. 2017, ApJ, 851, L30

Kilpatrick, C. D., \& Foley, R. J. 2018, ArXiv e-prints, arXiv:1806.00348

Liu, W., \& Dalgarno, A. 1995, ApJ, 454, 472

Liu, W., Dalgarno, A., \& Lepp, S. 1992, ApJ, 396, 679

Matsuura, M., Dwek, E., Meixner, M., et al. 2011, Science, 333, 1258

Matsuura, M., Dwek, E., Barlow, M. J., et al. 2015, ApJ, 800, 50

Nozawa, T., Kozasa, T., Umeda, H., Maeda, K., \& Nomoto, K. 2003, ApJ, 598, 785

Rho, J., Onaka, T., Cami, J., \& Reach, W. T. 2012, ApJ, 747, L6
Rho, J., Kozasa, T., Reach, W. T., et al. 2008, ApJ, 673, 271

Rho, J., Gomez, H. L., Boogert, A., et al. 2018, MNRAS, arXiv: 1707.08230

Sarangi, A., \& Cherchneff, I. 2013, ApJ, 776, 107

-. 2015, A\&A, 575, A95

Sarangi, A., Dwek, E., \& Arendt, R. G. 2018, ArXiv e-prints, arXiv:1804.06878

Schneider, R., Ferrara, A., \& Salvaterra, R. 2004, MNRAS, 351, 1379

Spitzer, L. 1978, Physical processes in the interstellar medium, doi:10.1002/9783527617722

Temim, T., \& Dwek, E. 2013, ApJ, 774, 8

Temim, T., Dwek, E., Arendt, R. G., et al. 2017, ApJ, 836, 129

Temim, T., Sonneborn, G., Dwek, E., et al. 2012, ApJ, 753, 72

Todini, P., \& Ferrara, A. 2001, MNRAS, 325, 726

Tsvetkov, D. Y., Shugarov, S. Y., Volkov, I. M., et al. 2018, ArXiv e-prints, arXiv:1801.00340

van Dyk, S. D., Filippenko, A. V., Fox, O. D., et al. 2017, The Astronomer's Telegram, 10378

Watson, D., Christensen, L., Knudsen, K. K., et al. 2015, Nature, 519,327

Xiang, D., Rui, L., Wang, X., et al. 2017, The Astronomer's Telegram, 10376

Zanardo, G., Staveley-Smith, L., Indebetouw, R., et al. 2014 , ApJ, 796, 82

Zhukovska, S., Henning, T., \& Dobbs, C. 2018, ApJ, 857, 94 